\title{
PROJECTIVE EMBEDDINGS OF PROJECTIVE SCHEMES BLOWN UP AT SUBSCHEMES
}

\author{
HUY TÀI HÀ
}

\begin{abstract}
Suppose $X$ is a nonsingular arithmetically Cohen-Macaulay projective scheme, $Z$ a nonsingular closed subscheme of $X$. Let $\tilde{X}$ be the blowup of $X$ centered at $Z, E_{0}$ the pull-back of a general hyperplane in $X$, and $E$ the exceptional divisor. In this paper, we study projective embeddings of $\tilde{X}$ given by the divisors $\mathcal{D}_{e, t}=t E_{0}-e E$. We give explicit values of $d$ and $\delta$ such that for all $e>0$ and $t>e d+\delta, \mathcal{D}_{e, t}$ embeds $\tilde{X}$ as a projectively normal and arithmetically Cohen-Macaulay scheme. We also give a uniform bound for the regularities of the ideal sheaves of these embeddings, and study their asymptotic behaviour as $t$ gets large compared to $e$. When $X$ is a surface and $Z$ is a 0 -dimensional subscheme, we further show that these embeddings possess property $N_{p}$ for all $t \gg e>0$.
\end{abstract}

Dedicated to the sixtieth birthday of Prof. A.V. Geramita

\section{INTRODUCTION}

Suppose $R$ is a Cohen-Macaulay standard graded $k$-algebra, and $X=\operatorname{Proj} R$ is a nonsingular projective scheme. Suppose $Z$ is a nonsingular closed subscheme of $X, I \subseteq R$ its defining ideal ( $I$ saturated). Let $\pi: \tilde{X} \rightarrow X$ be the blowup of $X$ centered at $Z$. For positive integers $e, t \in \mathbb{N}$, consider the divisor $\mathcal{D}_{e, t}=t E_{0}-e E$ on $\tilde{X}$, where $E_{0}$ is the pull-back to $\tilde{X}$ of a general hyperplane in $X$ and $E$ is the exceptional divisor of the blowing up. $\mathcal{D}_{e, t}$ corresponds to the linear system of hypersurfaces of degree $t$ containing $Z$ with multiplicity at least $e$. For $t \gg e>0$, it is known (cf. [3, Lemma 1.1]) that $\mathcal{D}_{e, t}$ is very ample on $\tilde{X}$. This gives a projective embedding $\tilde{X} \hookrightarrow \mathbb{P}^{N_{e, t}}$. Let $\mathcal{V}_{e, t}$ be the image of $\tilde{X}$ in this embedding (i.e. $\tilde{X} \stackrel{\sim}{\rightarrow} \mathcal{V}_{e, t} \subseteq \mathbb{P}^{N_{e, t}}$ ). In the last fifteen years, there have been many studies on these projective embeddings of $\tilde{X}$ in various situations depending upon the scheme $X$, its subscheme $Z$, and the values of $e$ and $t$. For instance, [3], [8], [9], [10], [11], [12], [15], [16], [18] and [19]. This line of works also has a close relation with the studies of diagonal subalgebras of a bi-graded algebra, such as in [2], [24], [26] and [27]. In this paper, we push the study on projective embeddings of blowup schemes a step forward. We will study

Key words and phrases. blowing up, Cohen-Macaulay variety, projective embedding. 2000 Mathematical Subject Classification. 14E25, 14M05, 13H10. 
the Cohen-Macaulayness, the regularity and syzygies of $\mathcal{V}_{e, t}$. We also investigate how these properties behave asymptotically (i.e. when $t \gg e>0$ ).

It has been shown by [3] (see also [26]) that under certain conditions, there exists an $f$ such that for all $e>0$ and $t>f e$, the embedding $\mathcal{V}_{e, t}$ of $\tilde{X}$ is arithmetically Cohen-Macaulay, i.e. it has a Cohen-Macaulay coordinate ring. However, no explicit bound for $f$ could be found unless one is in special situations, for example if it is known that the Rees algebra $\mathcal{R}(I)$ of $I$ is Cohen-Macaulay (see [26]), or when char $k=0, X=\mathbb{P}^{n}, Z$ is a scheme of fat points in $X$ and $e=1$ (see [10, Theorem 2.4]). In the first section of this paper, we address this problem again, and generalize the method of [10] to give an explicit bound for $f$ for any $X$ and $Z$, when the characteristic of $k$ is 0 . We give a constant $\delta=\delta(I)>0$ such that for all $e>0$ and $t>e d+\delta$ (where $d=d(I)$ is the maximum degree of a minimal system of homogeneous generators of $I$ ), the projective embedding $\mathcal{V}_{e, t}$ of $\tilde{X}$ is arithmetically CohenMacaulay (Theorem 1.3). An explicit bound for $f$ then can be taken to be $d+\delta$. Our result is stated as follows.

Theorem 0.1 (Theorem 1.3). For $t>e d+\delta$, the projective embedding $\mathcal{V}_{e, t}$ of $\tilde{X}$ is arithmetically Cohen-Macaulay.

In the second section of this paper, we study the regularity of the ideal sheaf and syzygies of $\mathcal{V}_{e, t}$. We prove that under a mild condition, the regularity of $\mathcal{V}_{e, t}$ is always bounded above by the dimension of $R$, when $t>e d+\delta$ (Theorem 2.1). This gives a uniform bound on the shifts of the minimal free resolution of the ideal sheaf of $\mathcal{V}_{e, t}$. A very interesting asymptotic behaviour is that the regularity of the ideal sheaf of $\mathcal{V}_{e, t}$ stablizes as $t$ gets large compared to $e$, if $e \geq$ ht $I-1$. We also prove this fact in Theorem 2.1.

Theorem 0.2 (Theorem 2.1). Let $\mathcal{J}_{e, t}$ be the ideal sheaf of $\mathcal{V}_{e, t}$. Suppose $H^{0}\left(\tilde{X}, w_{\tilde{X}}\right)=0$, where $w_{\tilde{X}}$ is the dualizing sheaf on $\tilde{X}$. Then,

$$
\text { reg } \mathcal{J}_{e, t} \leq n+1, \forall t>e d+\delta .
$$

If in addition, $X$ has a non-trivial dualizing sheaf $w_{X}$, and $e \geq h t I-1$, then for all $t \gg e$,

$$
\text { reg } \mathcal{J}_{e, t}=n+1 \text {. }
$$

Here, $n=\operatorname{dim} X$ (i.e. $n+1=\operatorname{dim} R$ ).

We further restrict our attention to the case when $X$ is a surface and $Z$ is a 0 -dimensional subscheme of $X$, and show that for any $p \in \mathbb{N}$, the embedding $\mathcal{V}_{e, t}$ possesses property $N_{p}$ for all $t \gg e>0$ (Theorem 2.2). This is a property, introduced by Green and Lazarsfeld ([13], [14]), that encodes a lot of information about the scheme. 
Results in this paper exhibit and support the philosophy that when embedding a variety into large projective spaces, it seems to have nicer algebraic properties, and its algebraic invariants seem to stablize as the dimension of the ambient space gets large. This philosophy is the guideline of many recent studies since the impact of works of Mumford [21], its amplification by St. Donat [25], and the strong theorems and conjectures of Green [13] and Green and Lazarsfeld [14.

Throughout this paper, our base field $k$ will be algebraically closed and of characteristic 0 .

\section{Cohen-Macaulayness}

Let us briefly recall our notations and terminologies. Unexplained terminologies follow from that of [6] and [17]. Let $R$ be a Cohen-Macaulay standard graded $k$-algebra of dimension $(\mathrm{n}+1), X=\operatorname{Proj} R$ a nonsingular projective scheme. Let $Z$ be a nonsingular closed subscheme of $X$ defined by a homogeneous ideal $I \subseteq R$ of positive height ( $I$ saturated). Let $\tilde{I}$ be the associated sheaf of $I$ on $X$, and let

$$
\pi: \tilde{X}=\operatorname{Proj}\left(\oplus_{n \geq 0} \tilde{I}^{n}\right) \rightarrow X
$$

be the blowup of $X$ centered at $Z$. Let $E$ be the exceptional divisor of this blowing up, and $E_{0}$ the pull-back to $\tilde{X}$ of a general hyperplane in $X$. For each pair of positive integers $e$ and $t$, consider the divisor $\mathcal{D}_{e, t}=t E_{0}-e E$ on $\tilde{X}$. Suppose $I$ is generated in degrees at most $d=d(I)$. By [3, Lemma 1.1], for each $e>0$ and $t \geq d e+1$, the divisor $\mathcal{D}_{e, t}$ is very ample on $\tilde{X}$, so from now on, we only consider values of $e>0$ and $t$ such that $t \geq d e+1$. For such values of $e$ and $t$, let $\tilde{X} \stackrel{\sim}{\rightarrow} \mathcal{V}_{e, t} \subseteq \mathbb{P}^{N_{e, t}}$ be the projective embedding of $\tilde{X}$ given by $\mathcal{D}_{e, t}$, where $N_{e, t}=\operatorname{dim}_{k} H^{0}\left(\tilde{X}, \mathcal{D}_{e, t}\right)-1$. We also denote by $r$ the height of $I$.

Suppose now that $I$ is generated by $f_{1}, \ldots, f_{m}$ where the degree of $f_{j}$ is $d_{j}$ for all $j=$ $1, \ldots, m$. Let $\mathcal{R}(I)=\oplus_{n \geq 0} I^{n} t^{n}$ be the Rees algebra of $I$. Let $S=R\left[y_{1}, \ldots, y_{m}\right]$, then $S$ has a natural bi-gradation (induced by $\operatorname{deg} F=(\gamma, 0)$ for any homogeneous polynomial $F \in R$ of degree $\gamma$, and $\operatorname{deg} y_{j}=\left(d_{j}, 1\right)$ for all $\left.j=1, \ldots, m\right)$. Consider the homomorphism

$$
S \rightarrow \mathcal{R}(I)
$$

given by the identity on $R$ and by sending $y_{j}$ to $f_{j} t$ for all $j=1, \ldots, m$. It is clear that this homomorphism is surjective and gives $\mathcal{R}(I)$ the structure of a bi-graded module over S. Suppose

$$
0 \rightarrow \ldots \rightarrow \oplus j S\left(-a_{i j},-b_{i j}\right) \rightarrow \ldots \rightarrow \oplus_{j} S\left(-a_{0 j},-b_{0 j}\right) \rightarrow \mathcal{R}(I) \rightarrow 0
$$


is the bi-graded minimal free resolution of $\mathcal{R}(I)$ over $S$. This minimal free resolution exists and is finite due to Hilbert's syzygy theorem. Let

$$
c_{i}=\max _{j}\left\{a_{i j}-b_{i j} d(I)\right\} .
$$

Clearly, $\max _{i}\left\{c_{i}-i\right\}$ is an invariant of $I$. We denote this invariant by $c(I)$.

Define $\delta=\delta(I)=\max \{c(I), 1\}$.

The following proposition of an extension of [10, Proposition 2.2].

Proposition 1.1. For $t>e d+\delta$, the projective embedding $\mathcal{V}_{e, t}$ of $\tilde{X}$ is projectively normal.

Proof. Suppose $t>e d+\delta$. By [3, Lemma 1.1], $\mathcal{D}_{e, t}$ is very ample on $\tilde{X}$. Let $S_{e, t}=$ $\operatorname{Sym}^{*}\left(H^{0}\left(\tilde{X}, \mathcal{D}_{e, t}\right)\right)$ be the coordinate ring of $\mathbb{P}^{N}=\mathbb{P}^{N_{e, t}}$ into which $\tilde{X}$ is embedded by $\mathcal{D}_{e, t}$, and let $I_{e, t} \subseteq S_{e, t}$ be the defining ideal of $\mathcal{V}_{e, t}$ in $\mathbb{P}^{N}$. One has the following exact sequence

$$
0 \rightarrow I_{e, t} \rightarrow S_{e, t} \rightarrow \oplus_{h \geq 0} H^{0}\left(\tilde{X}, \mathcal{O}_{\tilde{X}}\left(h \mathcal{D}_{e, t}\right)\right) \rightarrow \oplus_{h \geq 0} H^{1}\left(\mathbb{P}^{N}, \mathcal{J}_{e, t}(h)\right) \rightarrow 0,
$$

where $\mathcal{J}_{e, t}$ is the ideal sheaf of $\mathcal{V}_{e, t}$ in $\mathbb{P}^{N}$.

To prove that $\mathcal{V}_{e, t}$ is projectively normal, it is enough to show that for each $h \geq 0$, there is an isomorphism of $k$-vector spaces

$$
\left(\frac{S_{e, t}}{I_{e, t}}\right)_{h} \simeq H^{0}\left(\tilde{X}, \mathcal{O}_{\tilde{X}}\left(h \mathcal{D}_{e, t}\right)\right)
$$

This is clear for $h=0$. Suppose $h \geq 1$. It can be observed that a hyperplane in $\mathbb{P}^{N}$ when restricted to $\mathcal{V}_{e, t}$ pulls back to a hypersurface of degree $t$ in $X$ containing $Z$ with multiplicity at least $e$. Thus,

$$
\left(\frac{S_{e, t}}{I_{e, t}}\right)_{h} \simeq\left[\left(I_{t}^{e}\right)^{h}\right]_{h t}=\left[\left(I^{e}\right)^{h}\right]_{h t}=\left(I^{h e}\right)_{h t}
$$

Let $\mathcal{M}=\tilde{I} \cdot \mathcal{O}_{\tilde{X}}=\mathcal{O}_{\tilde{X}}(-E)$, then

$$
\mathcal{O}_{\tilde{X}}\left(h \mathcal{D}_{e, t}\right)=\mathcal{M}^{h e} \otimes \mathcal{O}_{\tilde{X}}\left(h t E_{0}\right)=\mathcal{M}^{h e} \otimes \pi^{*} \mathcal{O}_{X}(h t) .
$$

By [20, Proposition 10.2], we also have $\pi_{*} \mathcal{M}^{h e}=\tilde{I}^{h e}$. Hence, by the projection formula, we have

$$
\pi_{*} \mathcal{O}_{\tilde{X}}\left(h \mathcal{D}_{e, t}\right)=\pi_{*}\left(\mathcal{M}^{h e}\right) \otimes \mathcal{O}_{X}(h t)=\tilde{I}^{h e}(h t) .
$$

Thus,

$$
H^{0}\left(\tilde{X}, \mathcal{O}_{\tilde{X}}\left(h \mathcal{D}_{e, t}\right)\right)=H^{0}\left(X, \pi_{*} \mathcal{O}_{\tilde{X}}\left(h \mathcal{D}_{e, t}\right)\right)=H^{0}\left(X, \tilde{I}^{h e}(h t)\right)=\operatorname{dim}_{k} I_{h t}^{(h e)},
$$

where $I^{(h e)}$ is the saturation ideal of $I^{h e}$. 
Since $R$ is a standard graded $k$-algebra (i.e. $R$ is generated as a $k$-algebra by $R_{1}$ ), it follows from the argument of [4] that

$$
\operatorname{reg} I^{l} \leq l d+\delta \text { for any } l \geq 1
$$

(here, for an ideal $J$, reg $J$ is the regularity of $J$ ). Thus, for $t>e d+\delta$, we have

$$
h t>h e d+h \delta \geq h e d+\delta \geq \operatorname{reg} I^{h e} \geq \operatorname{sat}\left(I^{h e}\right),
$$

where sat $\left(I^{h e}\right)$ is the saturation degree of $I^{\text {he }}$ (i.e. the least degree starts from which $I^{\text {he }}$ and its saturation ideal $I^{(h e)}$ agree). Therefore, for $t>e d+\delta$, we have

$$
\operatorname{dim}_{k}\left[I^{h e}\right]_{h t}=\operatorname{dim}_{k} I_{h t}^{(h e)} .
$$

This together with (1.1) and (1.2) prove the proposition.

The following lemma plays an important role in the study of arithmetically Cohen-Macaulayness of $\mathcal{V}_{e, t}$.

Lemma 1.2. For $e>0, t \geq d e+1$, we have

(1) $R^{j} \pi_{*} \mathcal{O}_{\tilde{X}}\left(h \mathcal{D}_{e, t}\right)=0$ for all $j>0$ and $h \geq 0$.

(2) $H^{i}\left(\tilde{X}, \mathcal{O}_{\tilde{X}}\left(h \mathcal{D}_{e, t}\right)\right)=H^{i}\left(X, \tilde{I}^{h e}(h t)\right)$ for all $i \geq 0$ and $h \geq 0$.

Here, $\mathcal{O}_{\tilde{X}}$ and $w_{\tilde{X}}$ are the structure sheaf and the dualizing sheaf on $\tilde{X}$.

Proof. Again, we let $\mathcal{M}=\tilde{I} \mathcal{O}_{\tilde{X}}=\mathcal{O}_{\tilde{X}}(-E)$.

(1) For each $l \geq 0$, we have

$$
\mathcal{O}_{\tilde{X}}\left(h \mathcal{D}_{e, t}\right) \otimes \pi^{*} \mathcal{O}_{X}(l)=\mathcal{O}_{\tilde{X}}\left(h\left(t E_{0}-e E\right)\right) \otimes \pi^{*} \mathcal{O}_{X}(l)=\mathcal{M}^{h e} \otimes \pi^{*} \mathcal{O}_{X}(h t+l) .
$$

Now we will use the argument of [3, Proposition 1.5] to get

$$
H^{i}\left(\tilde{X}, \mathcal{O}_{\tilde{X}}\left(h \mathcal{D}_{e, t}\right) \otimes \pi^{*} \mathcal{O}_{X}(l)\right)=0, \forall i>0, l \gg 0 .
$$

Indeed, let $w_{X}$ be the dualizing sheaf on $X$. Since the height of $I$ is $r, w_{\tilde{X}}=\pi^{*} w_{X} \otimes \mathcal{M}^{1-r}$. Thus,

$$
\mathcal{M}^{h e} \otimes \pi^{*} \mathcal{O}_{X}(h t+l)=\mathcal{M}^{h e+r-1} \otimes \pi^{*} \mathcal{O}_{X}(h t+l) \otimes\left(\pi^{*} w_{X}\right)^{-1} \otimes w_{\tilde{X}}
$$

Observe that for any $c, d \in \mathbb{N}, \mathcal{M}^{d} \otimes \pi^{*} \mathcal{O}_{X}(c)=I_{c}^{d} \cdot \mathcal{O}_{\tilde{X}}$. It is known that $w_{X}^{-1}(g)$ is very ample on $X$ for some $g>0$. Thus, by [3, Lemma 1.1], for $l>d(h e+r-1)+g-h t$ (i.e. $h t+l>d(h e+r-1)+g)$, the divisor $\mathcal{M}^{h e+r-1} \otimes \pi^{*} \mathcal{O}_{X}(h t+l) \otimes\left(\pi^{*} w_{X}\right)^{-1}$ is very ample on $\tilde{X}$. By Kodaira's vanishing theorem, one has for $l>d(e+r-1)+g-h t$ and $i>0$,

$$
H^{i}\left(\tilde{X}, \mathcal{O}_{\tilde{X}}\left(h \mathcal{D}_{e, t}\right) \otimes \pi^{*} \mathcal{O}_{X}(l)\right)=0 .
$$


Next, for each $i>0$ and $h \geq 0$, since $\mathcal{O}_{X}(1)$ is very ample on $X$, by Serre's theorem, there exists an integer $l_{i, h}$ such that for all $l \geq l_{i, h}, p>0$ and $q \leq i$,

$$
H^{p}\left(X, R^{q} \pi_{*} \mathcal{O}_{\tilde{X}}\left(h \mathcal{D}_{e, t}\right) \otimes \mathcal{O}_{X}(l)\right)=0 .
$$

By the projection formula, we then have, for all $l \geq l_{i, h}, p>0$ and $q \leq i$,

$$
H^{p}\left(X, R^{q} \pi_{*}\left(\mathcal{O}_{\tilde{X}}\left(h \mathcal{D}_{e, t}\right) \otimes \pi^{*} \mathcal{O}_{X}(l)\right)\right)=0
$$

Consider the Leray spectral sequence

$$
E_{2}^{p, q}=H^{p}\left(X, R^{q} \pi_{*}\left(\mathcal{O}_{\tilde{X}}\left(h \mathcal{D}_{e, t}\right) \otimes \pi^{*} \mathcal{O}_{X}(l)\right)\right) \Rightarrow H^{p+q}\left(\tilde{X}, \mathcal{O}_{\tilde{X}}\left(h \mathcal{D}_{e, t}\right) \otimes \pi^{*} \mathcal{O}_{X}(l)\right) .
$$

By (1.4), this Leray spectral sequence (when used to compute $H^{i}\left(\tilde{X}, \mathcal{O}_{\tilde{X}}\left(h \mathcal{D}_{e, t}\right) \otimes \pi^{*} \mathcal{O}_{X}(l)\right)$ ) concentrates on its vertical boundary. This and (1.3) imply that, for all $l>\max \left\{l_{i, h}, d(h e+\right.$ $r-1)+g\}$ and all $i>0$,

$$
\Gamma\left(X, R^{i} \pi_{*}\left(\mathcal{O}_{\tilde{X}}\left(h \mathcal{D}_{e, t}\right) \otimes \pi^{*} \mathcal{O}_{X}(l)\right)\right)=H^{i}\left(\tilde{X}, \mathcal{O}_{\tilde{X}}\left(h \mathcal{D}_{e, t}\right) \otimes \pi^{*} \mathcal{O}_{X}(l)\right)=0 .
$$

Since $\mathcal{O}_{X}(1)$ is very ample on $X$, we also know that for $l \gg 0$,

$$
R^{i} \pi_{*}\left(\mathcal{O}_{\tilde{X}}\left(h \mathcal{D}_{e, t}\right) \otimes \pi^{*} \mathcal{O}_{X}(l)\right)=R^{i} \pi_{*} \mathcal{O}_{\tilde{X}}\left(h \mathcal{D}_{e, t}\right) \otimes \mathcal{O}_{X}(l)
$$

is generated by global sections. Thus, (1.5) implies $R^{i} \pi_{*}\left(\mathcal{O}_{\tilde{X}}\left(h \mathcal{D}_{e, t}\right)\right)=0 \forall i>0$. The result is proved.

(2) It follows from the Leray spectral sequence

$$
E_{2}^{p, q}=H^{p}\left(X, R^{q} \pi_{*} \mathcal{O}_{\tilde{X}}\left(h \mathcal{D}_{e, t}\right)\right) \Rightarrow H^{p+q}\left(\tilde{X}, \mathcal{O}_{\tilde{X}}\left(h \mathcal{D}_{e, t}\right)\right),
$$

and the result in part (1) that

$$
H^{i}\left(X, \pi_{*} \mathcal{O}_{\tilde{X}}\left(h \mathcal{D}_{e, t}\right)\right)=H^{i}\left(\tilde{X}, \mathcal{O}_{\tilde{X}}\left(h \mathcal{D}_{e, t}\right)\right) \forall i \geq 0 \text { and } h \geq 0
$$

We also have, by the projection formula and [20, Proposition 10.2],

$$
\pi_{*} \mathcal{O}_{\tilde{X}}\left(h \mathcal{D}_{e, t}\right)=\pi_{*}\left(\mathcal{M}^{h e} \otimes \pi^{*} \mathcal{O}_{X}(h t)\right)=\pi_{*} \mathcal{M}^{h e} \otimes \mathcal{O}_{X}(h t)=\tilde{I}^{h e}(h t) .
$$

Thus, the result follows, and the lemma is proved.

The main result of this section is stated as follows.

Theorem 1.3. For $t>e d+\delta$, the projective embedding $\mathcal{V}_{e, t}$ of $\tilde{X}$ is arithmetically CohenMacaulay.

Proof. Having Lemma 1.2, the proof now follows in the same line as that of 10, Theorem $2.4]$ with a slight modification. 
Suppose $t>e d+\delta$. As in Proposition 1.1, we let $S_{e, t}=\operatorname{Sym}^{*} H^{0}\left(\tilde{X}, \mathcal{D}_{e, t}\right)$ be the coordinate ring of $\mathbb{P}^{N}=\mathbb{P}^{N_{e, t}}$, and let $I_{e, t} \subseteq S_{e, t}$ be the defining ideal of $\mathcal{V}_{e, t}$ in $\mathbb{P}^{N}$. Since $\operatorname{dim} \mathcal{V}_{e, t}=$ $\operatorname{dim} X=n$, to prove that $\mathcal{V}_{e, t}$ is arithmetically Cohen-Macaulay, it is enough to show that $H^{1}\left(\mathbb{P}^{N}, \mathcal{J}_{e, t}(h)\right)=0$ for all $h \in \mathbb{Z}$ and $H^{i}\left(\mathbb{P}^{N}, \mathcal{O}_{\mathcal{V}_{e, t}}(h)\right)=0$ for all $i=1, \ldots, n-1$ and for all $h \in \mathbb{Z}$ (here, $\mathcal{J}_{e, t}$ is the ideal sheaf of $\mathcal{V}_{e, t}$ in $\mathbb{P}^{N}$ ).

By Proposition 1.1, we know that $H^{1}\left(\mathbb{P}^{N}, \mathcal{J}_{e, t}(h)\right)=0$ for all $h \geq 0$. For $h<0$, we see that $\mathcal{O}_{\mathcal{V}_{e, t}}(-h)$ is a very ample invertible sheaf on $\mathcal{V}_{e, t}$. Therefore, by Kodaira's vanishing theorem, we get $H^{0}\left(\mathbb{P}^{N}, \mathcal{O}_{\mathcal{V}_{e, t}}(h)\right)=0$ for all $h<0$. This implies that $H^{1}\left(\mathbb{P}^{N}, \mathcal{J}_{e, t}(h)\right)=0$ for all $h<0$. Thus,

$$
H^{1}\left(\mathbb{P}^{N}, \mathcal{J}_{e, t}(h)\right)=0, \text { for all } h \in \mathbb{Z}
$$

Let us now consider $H^{i}\left(\mathbb{P}^{N}, \mathcal{O}_{\mathcal{V}_{e, t}}(h)\right)(i=1, \ldots, n-1)$ for $h \geq 0$. It follows from Lemma 1.2 that, for all $i=1, \ldots, n-1$,

$$
H^{i}\left(\mathbb{P}^{N}, \mathcal{O}_{\mathcal{V}_{e, t}}(h)\right)=H^{i}\left(\tilde{X}, \mathcal{O}_{\tilde{X}}\left(h \mathcal{D}_{e, t}\right)\right)=H^{i}\left(X, \tilde{I}^{h e}(h t)\right) .
$$

Again, it follows from [4] that for $h>0$,

$$
\operatorname{reg} \tilde{I}^{h e} \leq h e d+\delta \leq h e d+h \delta<h t .
$$

Thus, $H^{i}\left(X, \tilde{I}^{h e}(h t)\right)=0$ for all $i=1, \ldots, n-1$. We also have $H^{i}\left(X, \mathcal{O}_{X}\right)=0$ for all $i=1, \ldots, n-1$ from the fact that $R$ is a Cohen-Macaulay ring. Therefore,

$$
H^{i}\left(\mathbb{P}^{N}, \mathcal{O}_{\mathcal{V}_{e, t}}(h)\right)=0,
$$

for all $i=1, \ldots, n-1$ and $h \geq 0$.

For $h<0$, we can again use the Kodaira's vanishing theorem to obtain

$$
H^{i}\left(\mathbb{P}^{N}, \mathcal{O}_{\mathcal{V}_{e, t}}(h)\right)=0,
$$

for all $i=1, \ldots, n-1$. This is due to the fact that $\mathcal{O}_{\mathcal{V}_{e, t}}(-h)$ is a very ample invertible sheaf on $\mathcal{V}_{e, t}$ for all $h<0$. The theorem is proved.

Remark: We should point out that with exactly the same proves all our results in this section are true even if $Z$ is only a locally complete intersection subscheme of $X$.

Corollary 1.3.1. Suppose $I$ is generated by s elements and the Rees algebra $\mathcal{R}(I)$ of $I$ is Cohen-Macaulay. Then, for all $e>0$ and $t>e d+(s-1)(d-1)$, the projective embedding $\mathcal{V}_{e, t}$ of $\tilde{X}$ is arithmetically Cohen-Macaulay. 
Proof. It follows from [26, Proposition 4.1] that, in the minimal free resolution of the Rees algebra $\mathcal{R}(I)$ of $I$, the bi-graded Betti numbers satisfy

$$
a_{i j} \leq s d-(s-1)+i .
$$

Thus, $\delta(I) \leq(s-1)[d(I)-1]$. The result now follows from Theorem 1.3.

\section{Regularity and $N_{p}$ PROperty}

In this section, we study how the regularity and the syzygies of $\mathcal{V}_{e, t}$ behave asymptotically. We use the same notations and terminologies as in the previous section.

Let us also recall the notions of Koszul complex and Koszul cohomologies which were studied in $[13]$.

Let $Y$ be a projective scheme. Let $\mathcal{L}$ be a very ample line bundle and $\mathcal{F}$ a coherent sheaf on $Y$. Let $W=H^{0}(Y, \mathcal{L})$ and $S=\operatorname{Sym}^{*} W$. Then, $S$ is the homogeneous coordinate ring of $\mathbb{P}(W)$, the projective space into which $\mathcal{L}$ embeds $Y$. Let $B=B(\mathcal{F}, \mathcal{L})=\oplus_{q \in \mathbb{Z}} H^{0}(Y, \mathcal{F} \otimes$ $q \mathcal{L})=\oplus_{q \in \mathbb{Z}} B_{q}$ a $S$-graded module.

Definition. The Koszul complex of $B$ is defined to be

$$
\ldots \rightarrow \wedge^{p+1} W \otimes B_{q-1} \stackrel{d_{p+1, q-1}}{\rightarrow} \wedge^{p} W \otimes B_{q} \stackrel{d_{p, q}}{\rightarrow} \wedge^{p-1} W \otimes B_{q+1} \rightarrow \ldots
$$

and the Koszul cohomology groups of $B$ are defined to be

$$
\mathcal{K}_{p, q}(\mathcal{F}, \mathcal{L})=\frac{\operatorname{ker} d_{p, q}}{\operatorname{im} d_{p+1, q-1}}, \text { for } p, q \in \mathbb{Z}
$$

When $\mathcal{L}=\mathcal{L}(D)$ is the invertible sheaf corresponding to a divisor $D$ and $\mathcal{F}=\tilde{M}$ is the sheaf associated to a module $M$, we write $\mathcal{K}_{p, q}(M, D)$ for $\mathcal{K}_{p, q}(\mathcal{F}, \mathcal{L})$. When $\mathcal{F}$ is the structure sheaf $\mathcal{O}_{Y}$, we also write $\mathcal{K}_{p, q}(\mathcal{L})$ for $\mathcal{K}_{p, q}(\mathcal{F}, \mathcal{L})$.

The following theorem gives an upper bound for the regularity of the ideal sheaf of $\mathcal{V}_{e, t}$, and shows that for almost all $e$, but a finite number of values, this regularity stablizes as $t \gg e$.

Theorem 2.1. Let $\mathcal{J}_{e, t}$ be the ideal sheaf of $\mathcal{V}_{e, t}$. Suppose $H^{0}\left(\tilde{X}, w_{\tilde{X}}\right)=0$, where $w_{\tilde{X}}$ is the dualizing sheaf on $\tilde{X}$. Then,

$$
\text { reg } \mathcal{J}_{e, t} \leq n+1, \forall t>e d+\delta .
$$

If in addition, $X$ has a non-trivial dualizing sheaf $w_{X}$, and $e \geq h t I-1$, then for all $t \gg e$,

$$
\operatorname{reg} \mathcal{J}_{e, t}=n+1
$$


Proof. Let $S_{e, t}$ and $I_{e, t}$ be as before (see Proposition 1.1). It follows from Proposition 1.1, and Theorem 1.3 that for $t>e d+\delta$,

$$
\frac{S_{e, t}}{I_{e, t}} \simeq \oplus_{h \geq 0} H^{0}\left(\tilde{X}, \mathcal{O}_{\tilde{X}}\left(h \mathcal{D}_{e, t}\right)\right)
$$

is a Cohen-Macaulay ring. Thus, by Green's syzygy theorem ([13, 1.b.4]), the minimal free resolution of $S_{e, t} / I_{e, t}$ is given by

$$
0 \rightarrow F_{N-n} \rightarrow \ldots \rightarrow F_{1} \rightarrow F_{0}=S_{e, t} \rightarrow S_{e, t} / I_{e, t} \rightarrow 0
$$

where

$$
F_{i}=\bigoplus_{q \geq 1} \mathcal{K}_{i, q}\left(\mathcal{D}_{e, t}\right) \otimes S(-i-q), \text { for } i=1, \ldots, N-n
$$

We first prove that reg $\mathcal{J}_{e, t} \leq n+1$ for all $t>e d+\delta$. This is equivalent to showing that $\mathcal{K}_{i, q}\left(\mathcal{D}_{e, t}\right)=0$ for all $1 \leq i \leq N-n$ and $q \geq n+1$, when $t>e d+\delta$. Suppose that $1 \leq i \leq N-n$ and $q \geq n+1$. Let $K_{\tilde{X}}$ be the canonical divisor of $\tilde{X}$, then $w_{\tilde{X}}$ is the sheaf associated to $K_{\tilde{X}}$ on $\tilde{X}$. Since $\mathcal{V}_{e, t}$ is arithmetically Cohen-Macaulay, it follows from Green's Duality theorem $(\sqrt{13}, 2 . c .6])$ that

$$
\mathcal{K}_{i, q}\left(\mathcal{D}_{e, t}\right)^{*} \simeq \mathcal{K}_{N-n-i, n+1-q}\left(K_{\tilde{X}}, \mathcal{D}_{e, t}\right), \forall q \geq 1 .
$$

By Green's Vanishing theorem $([13,3 . a .1])$, it suffices now to show that

$$
h^{0}\left(\tilde{X}, w_{\tilde{X}} \otimes \mathcal{O}_{\tilde{X}}\left((n+1-q) \mathcal{D}_{e, t}\right)\right) \leq N-n-i \forall t>e d+\delta .
$$

This is indeed true. If $q=n+1$, this follows from the hypothesis that $H^{0}\left(\tilde{X}, w_{\tilde{X}}\right)=0$. Otherwise, if $q>n+1$, then by Serre's duality one has $H^{0}\left(\tilde{X}, w_{\tilde{X}} \otimes \mathcal{O}_{\tilde{X}}\left((n+1-q) \mathcal{D}_{e, t}\right)\right)=$ $H^{n}\left(\tilde{X}, \mathcal{O}_{\tilde{X}}\left((q-n-1) \mathcal{D}_{e, t}\right)\right)$. Thus, by Lemma 1.2 and [20, Proposition 10.2], we have

$$
H^{0}\left(\tilde{X}, w_{\tilde{X}} \otimes \mathcal{O}_{\tilde{X}}\left((n+1-q) \mathcal{D}_{e, t}\right)\right)=H^{n}\left(X, \tilde{I}^{e(q-n-1)}(t(q-n-1))\right) .
$$

It follows from [4] that for $t>e d+\delta$,

$$
\operatorname{reg} \tilde{I}^{e(q-n-1)}<t(q-n-1)
$$

whence

$$
H^{n}\left(X, \tilde{I}^{e(q-n-1)}(t(q-n-1))\right)=0 .
$$

Therefore,

$$
H^{0}\left(\tilde{X}, w_{\tilde{X}} \otimes \mathcal{O}_{\tilde{X}}\left((n+1-q) \mathcal{D}_{e, t}\right)\right)=0 \forall t>e d+\delta,
$$

and (2.1) is proved.

Suppose now that $e \geq$ ht $I-1$. By Green's Duality theorem ([13, 2.c.6]) we also have

$$
\mathcal{K}_{N-n, n}\left(\mathcal{D}_{e, t}\right)^{*} \simeq \mathcal{K}_{0,1}\left(K_{\tilde{X}}, \mathcal{D}_{e, t}\right) .
$$


Let $W=H^{0}\left(\tilde{X}, \mathcal{O}_{\tilde{X}}\left(\mathcal{D}_{e, t}\right)\right)$, the Koszul complex of $\oplus_{h \geq 0} H^{0}\left(\tilde{X}, \mathcal{O}_{\tilde{X}}\left(h \mathcal{D}_{e, t}\right)\right)$ at degree $(0,1)$ is

$$
\wedge W \otimes H^{0}\left(\tilde{X}, w_{\tilde{X}}\right) \stackrel{d_{1,0}}{\rightarrow} W \otimes H^{0}\left(w_{\tilde{X}} \otimes \mathcal{O}_{\tilde{X}}\left(\mathcal{D}_{e, t}\right)\right) \stackrel{d_{0,1}}{\rightarrow} 0 .
$$

By the hypothesis, since $H^{0}\left(\tilde{X}, w_{\tilde{X}}\right)=0$, we have

$$
\mathcal{K}_{0,1}\left(K_{\tilde{X}}, \mathcal{D}_{e, t}\right)=W \otimes H^{0}\left(w_{\tilde{X}} \otimes \mathcal{O}_{\tilde{X}}\left(\mathcal{D}_{e, t}\right)\right) .
$$

Recall that $r$ is the height of $I, w_{X}$ is the dualizing sheaf on $X$, and $\mathcal{M}=\tilde{I} \mathcal{O}_{\tilde{X}}=\mathcal{O}_{\tilde{X}}(-E)$, we have

$$
H^{0}\left(\tilde{X}, w_{\tilde{X}} \otimes \mathcal{O}_{\tilde{X}}\left(\mathcal{D}_{e, t}\right)\right)=H^{0}\left(X, \pi_{*}\left(w_{\tilde{X}} \otimes \mathcal{O}_{\tilde{X}}\left(h \mathcal{D}_{e, t}\right)\right)\right)=H^{0}\left(\tilde{X}, \pi_{*}\left(\mathcal{M}^{e+1-r} \otimes \pi^{*} w_{X}(t)\right)\right) .
$$

For $e \geq r-1$, by the projection formula and by [20, Proposition 10.2], we now obtain

$$
H^{0}\left(\tilde{X}, w_{\tilde{X}} \otimes \mathcal{O}_{\tilde{X}}\left(\mathcal{D}_{e, t}\right)\right)=H^{0}\left(X, \tilde{I}^{e+1-r} \otimes w_{X} \otimes \mathcal{O}_{X}(t)\right) .
$$

It can be seen that $\mathcal{O}_{X}(1)$ is an ample invertible sheaf on $X$, so for $t \gg e \geq r-1$, $\tilde{I}^{e+1-r} \otimes w_{X} \otimes \mathcal{O}_{X}(t)$ is generated by its global sections. Therefore, since $\tilde{I}^{e+1-r} \otimes w_{X} \otimes \mathcal{O}_{X}(t)$ cannot be the zero sheaf on $X$, we must have $H^{0}\left(X, \tilde{I}^{e+1-r} \otimes w_{X} \otimes \mathcal{O}_{X}(t)\right) \neq 0$ for all $t \gg e \geq r-1$. It now follows from (2.2) and (2.3) that, for all $t \gg e \geq$ ht $I-1$,

$$
\mathcal{K}_{0,1}\left(K_{\tilde{X}}, \mathcal{D}_{e, t}\right) \neq 0 \text {, i.e. } \mathcal{K}_{N-n, n}\left(\mathcal{D}_{e, t}\right) \neq 0 .
$$

This implies that reg $J_{e, t} \geq n+1$. The result now follows. The theorem is proved.

Remark: It follows from the proof of Theorem 2.1 that even without the condition $H^{0}\left(\tilde{X}, w_{\tilde{X}}\right)=0$, we can still show that reg $\mathcal{J}_{e, t} \leq n+1$ for all $t \gg e>0$.

Before moving on to study the syzygies of $\mathcal{V}_{e, t}$, let us recall the notion of property $N_{p}$ (from [14]).

Definition. Let $Y$ be a projective variety and let $\mathcal{L}$ be a very ample line bundle on $Y$ defining an embedding $\varphi_{\mathcal{L}}: Y \hookrightarrow \mathbb{P}=\mathbb{P}\left(H^{0}(Y, \mathcal{L})^{*}\right)$.

(1) The line bundle $\mathcal{L}$, or the embedding $\varphi_{\mathcal{L}}(Y)$ of $Y$, is said to have property $N_{0}$ if $\varphi_{\mathcal{L}}(Y)$ is projectively normal, i.e. $\mathcal{L}$ is normally generated.

(2) Let $S=\operatorname{Sym}^{*} H^{0}(Y, \mathcal{L})$, the homogeneous coordinate ring of the projective space $\mathbb{P}$. Suppose $A$ is the homogeneous coordinate ring of $\varphi_{\mathcal{L}}(Y)$ in $\mathbb{P}$, and

$$
0 \rightarrow F_{n} \rightarrow F_{n-1} \rightarrow \ldots \rightarrow F_{0} \rightarrow A \rightarrow 0
$$


is a minimal free resolution of $A$. The line bundle $\mathcal{L}$, or the embedding $\varphi_{\mathcal{L}}(Y)$ of $Y$, is said to have property $N_{p}$ (for $p \in \mathbb{N}$ ) if and only if it has property $N_{0}, F_{0}=S$ and $F_{i}=S(-i-1)^{\alpha_{i}}$ with $\alpha_{i} \in \mathbb{N}$ for all $1 \leq i \leq p$.

In what comes next, we restrict our attention to the case when $X$ is a surface, i.e. $\operatorname{dim} R=3$, and $Z$ is a nonsingular 0 -dimensional subscheme of $X$. We will show that for any $p \in \mathbb{N}$, the projective embedding $\mathcal{V}_{e, t}$ of $\tilde{X}$ possesses property $N_{p}$ for all $t \gg e>0$. We apply the method which was demonstrated in [23].

Theorem 2.2. Suppose $X$ is a nonsingular surface, and $Z$ is a nonsingular 0-dimensional subscheme of $X$, where $\tilde{X}$ is the blowup of $X$ centered at $Z$. Then, for any $p \in \mathbb{N}$, the projective embedding $\mathcal{V}_{e, t}$ of $\tilde{X}$ possesses property $N_{p}$ for all $t \gg e>0$.

Proof. For $p=0$, the theorem is proved in Proposition 1.1. Suppose now that $p>0$. Let $S=S_{e, t}, I_{e, t}$ and $\mathcal{J}_{e, t}$ be as before. It follows from Theorem 2.1 and the remark after it that for all $t \gg e>0$,

$$
\operatorname{reg} \mathcal{J}_{e, t} \leq 3
$$

It also follows from Theorem 1.3 that for all $t \gg e>0, \mathcal{V}_{e, t}$ is arithmetically CohenMacaulay. Thus, for all $t \gg e>0$, the defining ideal $I_{e, t}$ of $\mathcal{V}_{e, t}$ has the following minimal free resolution

$$
\begin{aligned}
& S(-N)^{\beta_{N-2, N}} \quad S(-4)^{\beta_{2,4}} \quad S(-3)^{\beta_{1,3}} \\
& \bigoplus \quad \bigoplus \quad \bigoplus \\
& 0 \rightarrow S(-(N-1))^{\beta_{N-2, N-1}} \rightarrow \ldots \rightarrow S(-3)^{\beta_{2,3}} \rightarrow S(-2)^{\beta_{1,2}} \rightarrow I_{e, t} \rightarrow 0 \text {, }
\end{aligned}
$$

where $N=N_{e, t}=\operatorname{dim}_{k} H^{0}\left(\tilde{X}, \mathcal{D}_{e, t}\right)-1$. To show that $\mathcal{V}_{e, t}$ possesses property $N_{p}$, it is enough to show that $\beta_{i, i+2}=0$ for all $1 \leq i \leq p$.

Let $\mathcal{C}$ be a general hyperplane section of $\mathcal{V}_{e, t}$, then $\mathcal{C}$ is an arithmetically Cohen-Macaulay curve in $\mathbb{P}^{N-1}$ with the same minimal free resolution as that of $\mathcal{V}_{e, t}$. Let $A$ be the homogeneous coordinate ring of $\mathcal{C}$ in $\mathbb{P}^{N-1}$. Then, the Betti numbers of $\mathcal{C}$ (also of $\mathcal{V}_{e, t}$ ) are given by

$$
\beta_{i, j}=\operatorname{Tor}_{i}^{T}(A, k)_{j} \forall i, j
$$

where $T=k\left[x_{0}, \ldots, x_{N-1}\right]$ is the coordinate ring of $\mathbb{P}^{N-1}$. 
Now, let $i(E)=E . E$ be the self-intersection number of $E$. Let $A_{e, t}=S_{e, t} / I_{e, t}$ be the homogeneous coordinate ring of $\mathcal{V}_{e, t}$, and $\mathbb{H}_{\mathcal{V}_{e, t}}$ the Hilbert function of $\mathcal{V}_{e, t}$, i.e.

$$
\mathbb{H}_{\mathcal{V}_{e, t}}(\lambda)=\operatorname{dim}_{k}\left(A_{e, t}\right)_{\lambda}
$$

For $t \gg e>0$, since $\mathcal{V}_{e, t}$ is arithmetically Cohen-Macaulay, we have

$$
\operatorname{dim}_{k}\left(A_{e, t}\right)_{\lambda}=\operatorname{dim}_{k} H^{0}\left(\mathcal{V}_{e, t}, \mathcal{O}_{\mathcal{V}_{e, t}}(\lambda)\right)=\operatorname{dim}_{k} H^{0}\left(\tilde{X}, \mathcal{O}_{\tilde{X}}\left(\lambda \mathcal{D}_{e, t}\right)\right)
$$

Since $\mathcal{V}_{e, t}$ is arithmetically Cohen-Macaulay, we also have $H^{1}\left(\tilde{X}, \mathcal{O}_{\tilde{X}}\left(\lambda \mathcal{D}_{e, t}\right)\right)=0$ for all $\lambda \in \mathbb{Z}$. Thus, by the Riemann-Roch theorem, we have

$$
\operatorname{dim}_{k}\left(A_{e, t}\right)_{\lambda}=h^{0}\left(\tilde{X}, \lambda \mathcal{D}_{e, t}\right) \leq \frac{1}{2}\left[\left(\lambda \mathcal{D}_{e, t}\right)^{2}-\lambda \mathcal{D}_{e, t} \cdot K_{\tilde{X}}\right]+1+p_{\tilde{X}},
$$

where $K_{\tilde{X}}$ and $p_{\tilde{X}}$ are the canonical divisor and the arithmetic genus of $X$. Let $\delta_{e, t}$ be the degree of $\mathcal{V}_{e, t}$, then we now have

$$
\delta_{e, t}=\operatorname{deg} \mathcal{V}_{e, t} \leq\left(\mathcal{D}_{e, t}\right)^{2}=\left(t E_{0}-e E\right)^{2}=t^{2}+e^{2} i(E) .
$$

Let $\mathbb{X}=\mathcal{C} \cap H$ be a general hyperplane section of $\mathcal{C}$, then $\mathbb{X}$ is a set of $\delta_{e, t}$ points in $\mathbb{P}^{N-2}$. Let $\mathcal{J}_{C}$ be the ideal sheaf of $\mathcal{C}$ in $\mathbb{P}^{N-1}, \mathcal{J}_{\mathbb{X}}$ the ideal sheaf of $\mathbb{X}$ in $H \simeq \mathbb{P}^{N-2}$, and $\mathbb{H}_{\mathbb{X}}$ the Hilbert function of $\mathbb{X}$. Since $\mathcal{C}$ is arithmetically Cohen-Macaulay, it follows from the exact sequence

$$
0 \rightarrow \mathcal{J}_{C} \rightarrow \mathcal{J}_{C}(1) \rightarrow \mathcal{J}_{\mathbb{X}}(1) \rightarrow 0
$$

that

$$
0 \rightarrow H^{1}\left(\mathbb{P}^{N-2}, \mathcal{J}_{\mathbb{X}}(1)\right) \rightarrow H^{2}\left(\mathbb{P}^{N-1}, \mathcal{J}_{C}\right) \rightarrow H^{2}\left(\mathbb{P}^{N-1}, \mathcal{J}_{C}(1)\right) \rightarrow 0
$$

This implies

$$
h^{2}\left(\mathbb{P}^{N-1}, \mathcal{J}_{C}\right)-h^{2}\left(\mathbb{P}^{N-1}, \mathcal{J}_{C}(1)\right)=h^{1}\left(\mathbb{P}^{N-2}, \mathcal{J}_{\mathbb{X}}(1)\right)=\delta_{e, t}-\mathbb{H}_{\mathbb{X}}(1) .
$$

Let $Z_{e}$ be the subscheme of $X$ defined by $I^{e}$. Then, $Z_{e}$ is also a 0 -dimensional subscheme of $X$. We, therefore, know that the Hilbert function of $Z_{e}$ eventually stablizes at $\operatorname{deg} Z_{e}$. Since $R$ is standard graded $k$-algebra (i.e. $R$ is generated as a $k$-algebra by $R_{1}$ ) and $\operatorname{dim} R=3$, we have $\operatorname{dim}_{k} R_{t} \geq\left(\begin{array}{c}t+2 \\ 2\end{array}\right)$. Thus, for $t \gg e$,

$$
N=\operatorname{dim}_{k} R_{t}-\operatorname{deg} Z_{e} \geq\left(\begin{array}{c}
t+2 \\
2
\end{array}\right)-\operatorname{deg} Z_{e} .
$$

Clearly, for $t \gg e$ (more precisely, when $t \geq \frac{e^{2} i(E)+2 \operatorname{deg} Z_{e}+1+p}{3}$ ),

$$
2 N-3 \geq t^{2}+e^{2} i(E)+p \geq \delta_{e, t}+p .
$$

By 迆, we now have, for $t \gg e$,

$$
\mathbb{H}_{\mathbb{X}}(1) \geq \min \left\{\delta_{e, t}, N-1\right\} \geq \delta_{e, t}-(N-2)+p .
$$


(2.4) then implies

$$
h^{2}\left(\mathbb{P}^{N-1}, \mathcal{J}_{C}\right)-h^{2}\left(\mathbb{P}^{N-1}, \mathcal{J}_{C}(1)\right) \leq(N-2)-p<N-2 .
$$

By [22, Corollary 3.3], this only happens if $h^{2}\left(\mathbb{P}^{N-1}, \mathcal{J}_{C}(1)\right)=0$. Thus, for $t \gg e,(2.4)$ and (2.5) give

$$
h^{2}\left(\mathbb{P}^{N-1}, \mathcal{J}_{C}\right)=h^{1}\left(\mathbb{P}^{N-2}, \mathcal{J}_{\mathbb{X}}(1)\right)=\delta_{e, t}-\mathbb{H}_{\mathbb{X}}(1) \leq(N-2)-p
$$

Now, let $w_{A}$ be the canonical module of $A$. Then,

$$
\operatorname{dim}_{k}\left(w_{A}\right)_{0}=\operatorname{dim}_{k}\left[H_{\mathfrak{m}}^{2}(A)\right]_{0}=h^{1}\left(\mathbb{P}^{N-1}, \mathcal{O}_{\mathcal{C}}\right)=h^{2}\left(\mathbb{P}^{N-1}, \mathcal{J}_{C}\right) \leq(N-2)-p .
$$

Furthermore, by [23, Lemma 2.4], $w_{A}$ is torsion free as an $A$-module. Thus, the vanishing theorem of [7, Theorem 1.1] gives us, for $t \gg e$,

$$
\left[\operatorname{Tor}_{s}^{T}\left(w_{A}, k\right)\right]_{s}=0, \forall s \geq(N-2)-p .
$$

By duality, we now obtain, for $t \gg e$,

$$
\left[\operatorname{Tor}_{i}^{T}(A, k)\right]_{i+2}=0, \forall i \leq p,
$$

i.e. for $t \gg e$,

$$
\beta_{i, i+2}=0 \forall i \leq p
$$

This implies that $\mathcal{V}_{e, t}$ possesses property $N_{p}$. The theorem is proved.

Remark: It would be interesting to have an explicit bound $d_{e, p}$ such that for any $p \in \mathbb{N}$ and $e>0$, the projective embedding $\mathcal{V}_{e, t}$ of $\tilde{X}$ has property $N_{p}$ for all $t \geq d_{e, p}$. It follows from Theorem 1.3 and the proof of Theorem 2.2 that a bound could be given as

$$
d_{e, p}=\max \left\{e[d(I)+\delta(I)]+1, \frac{e^{2} i(E)+2 \operatorname{deg} Z_{e}+1+p}{3}\right\},
$$

i.e. for any $p \in \mathbb{N}$ and $e>0, \mathcal{V}_{e, t}$ has property $N_{p}$ for all

$$
t \geq \max \left\{e[d(I)+\delta(I)]+1, \frac{e^{2} i(E)+2 \operatorname{deg} Z_{e}+1+p}{3}\right\} .
$$

Acknowledgement. The author would like to thank Prof. S. D. Cutkosky for many useful discussions on topics related to the materials of this paper.

\section{REFERENCES}

[1] Ballico, E. (1989). On singular curves in positive characteristic. Math. Nachr. 141, 267-273.

[2] Conca, A., Herzog, J., Trung, N.V., and Valla, G. (1997). Diagonal subalgebras of bigraded algebras and embeddings of blow-ups of projective spaces. Amer. J. Maths., 119, 859-901.

[3] Cutkosky, S.D. and Herzog, J. (1997). Cohen-Macaulay coordinate rings of blowup schemes. Comment. Math. Helv. 72, 605-617. 
[4] Cutkosky, S.D., Herzog, J. and Trung, N.V. (1999). Asymptotic behaviour of the Castenuovo-Mumford regularity. Compositio Math. 118, 243-261.

[5] Cutkosky, S.D. and Hà, H. Tài. Arithmetic Macaulayfication of projective schemes. In preparation.

[6] Eisenbud, D. (1995). Commutative algebra with a view toward algebraic geometry. Graduate Text 150. Springer-Verlag. New York.

[7] Eisenbud, D. and Koh, J. (1991). Some linear syzygy conjectures. Adv. Math. 90, 47-76.

[8] Geramita, A.V. and Gimigliano, A. (1991). Generators for the defining ideal of certain rational surfaces. Duke Mathematical Journal, 62, no. 1, 61-83.

[9] Geramita, A.V., Gimigliano, A. and Harbourne, B. (1994). Projectively normal but superabundant embeddings of rational surfaces in projective space. J. Algebra. 169, no 3, 791-804.

[10] Geramita, A.V., Gimigliano, A. and Pitteloud, Y. (1995). Graded Betti numbers of some embedded rational $n$-folds. Math. Ann. 301, 363-380.

[11] Gimigliano, A. (1989). On Veronesean surfaces. Proc. Konin. Ned. Akad. van Wetenschappen, Ser. A, 92, 71-85.

[12] Gimigliano, A. and Lozenzini, A. (1993). On the ideal of veronesean surfaces. Can. J. Math. 43. 758-777.

[13] Green, M. (1984). Koszul cohomology and the geometry of projective varieties. J. Diff. Geom. 19, $125-171$.

[14] Green, M. and Lazarsfeld, R. (1988). Some results on the syzygies of finite sets and algebraic curves. Compositio Math. 67, 207-219.

[15] Hà, Huy Tài (2000). Rational surfaces from an algebraic perspective. PhD Thesis. Queen's University, Kingston, Canada.

[16] Hà, H. Tài (2002). Box-shaped matrices and the defining ideal of certain blowup surfaces. J. Pure. Appl. Algebra. 167, 203-224.

[17] Hartshorne, R. (1977). Algebraic Geometry. Grad text in Math. 52. Springer-Verlag. New York.

[18] Holay, S.H. (1996). Generators of ideals defining certain surfaces in projective space. Canadian J. Math. 48 (3), 585-595.

[19] Holay, S.H. (1996). Free resolutions of the defining ideal of certain rational surfaces. Manuscripta Mathematica. 90 (1), 23-37.

[20] Matsumura, H. (1959). Geometric structure of the cohomology rings in abstract algebraic geometry. Mem. Coll. Sci. Univ. Kyoto. (A). 32, 33-84.

[21] Mumford, D. (1969). Varieties defined by quadratic equations. C.I.M.E., III, 29-100.

[22] Nagel, U. (1992). On bounds for cohomological Hilbert functions. J. Algebra. 150, 231-244.

[23] Nagel, U. (1995). On the defining equations and syzygies of arithmetically Cohen-Macaulay varieties in arbitrary characteristic. J. Algebra. 175, 359-372.

[24] Simis, A., Trung, N.V. and Valla, G. (1998). The diagonal subalgebra of a blow-up algebra. J. Pure Appl. Algebra, 125, 305-328.

[25] Saint-Donat, B. (1972). Sur les équations définissant une courbe algébrique. C. R. Acad. Sci. Paris. 274, 324-327.

[26] Lavila-Vidal, O. (1998). On the Cohen-Macaulay property of diagonal subalgebras of the Rees algebra. Manuscripta Math. 95, no. 1, 47-58.

[27] Lavila-Vidal, O. (1999). On the diagonals of a Rees algebra. PhD Thesis. Universitat de Barcelona, Barcelona.

Department of Mathematics, University of Missouri, Columbia MO 65211

E-mail address: tai@math.missouri.edu 